ISSN 1855-3966 (printed edn.), ISSN 1855-3974 (electronic edn.)

\author{
ARS MATHEMATICA CONTEMPORANEA 15 (2018) 173-190 \\ https://doi.org/10.26493/1855-3974.1341.5a3 \\ (Also available at http://amc-journal.eu)
}

\title{
Groups of symmetric crosscap number less than or equal to 17
}

\author{
Adrián Bacelo * \\ Departamento de Álgebra, Facultad de Matemáticas, \\ Universidad Complutense, 28040 Madrid, Spain
}

Received 3 March 2017, accepted 21 March 2018, published online 13 June 2018

\begin{abstract}
Every finite group $G$ acts on some non-orientable unbordered surfaces. The minimal topological genus of those surfaces is called the symmetric crosscap number of $G$. It is known that 3 is not the symmetric crosscap number of any group but it remains unknown whether there are other such values, called gaps.

In this paper we obtain the groups with symmetric crosscap number less than or equal to 17 . Also, we obtain six infinite families with symmetric crosscap number of the form $12 k+3$.
\end{abstract}

Keywords: Symmetric crosscap number, Klein surfaces.

Math. Subj. Class.: 57M60, 20F05, 20H10, 30F50

\section{Introduction}

A Klein surface $X$ is a compact surface endowed with a dianalytic structure [1]. Klein surfaces may be seen as a generalization of Riemann surfaces including bordered and nonorientable surfaces. An orientable unbordered Klein surface is a Riemann surface. Given a Klein surface $X$ of topological genus $g$ with $k$ boundary components the number $p=$ $\eta g+k-1$ is called the algebraic genus of $X$, where $\eta=2$ if $X$ is an orientable surface and $\eta=1$ otherwise.

In the study of Klein surfaces and their automorphism groups the non-euclidean crystallographic (NEC) groups play an essential role. An NEC group $\Gamma$ is a discrete subgroup of $\mathcal{G}$ (the full group of isometries of the hyperbolic plane $\mathcal{H}$ ) with compact quotient $\mathcal{H} / \Gamma$. For a Klein surface $X$ with $p \geq 2$ there exists an NEC group $\Gamma$, such that $X=\mathcal{H} / \Gamma$, [27].

\footnotetext{
* The author wish to express his deep gratitude to the referees for their careful checking of the manuscript and for their very useful suggestions concerning both the style and the precision of arguments. The author is partially supported by UCM910444 and MTM2014-55565.

E-mail address: abacelo@ucm.es (Adrián Bacelo)
} 
A finite group $G$ of order $N$ is a subgroup of the automorphism group of a Klein surface $X=\mathcal{H} / \Gamma$ if and only if there exists an NEC group $\Lambda$ such that $\Gamma$ is a normal subgroup of $\Lambda$ with index $N$ and $G=\Lambda / \Gamma$. Every finite group $G$ acts as a subgroup of the automorphism group of some non-orientable surface without boundary, see [7]. The minimum topological genus of these surfaces is called the symmetric crosscap number of $G$ and it is denoted by $\tilde{\sigma}(G)$. Such a surface of topological genus $g \geq 3$ has at most $84(g-2)$ automorphisms. Hence, for each $g$ there is a finite number of groups acting on surfaces of genus $g$. The systematic study of the symmetric crosscap number was begun by May in [23], although previous results from other authors are also to be noted, see for instance [7, 14, 19].

Four types of inter-related problems arise naturally when dealing with the symmetric crosscap number $\tilde{\sigma}(G)$.

First of all, to obtain $\tilde{\sigma}(G)$ for any given group $G$, and for the groups belonging to a given infinite family.

Second, to obtain $\tilde{\sigma}(G)$ for all groups $G$ with $o(G)<n$ for a given (small) value of $n$.

Third, for a given value of $g$, to obtain all groups $G$ such that $\tilde{\sigma}(G)=g$. Evidently this question is feasible only for low values of $g$.

Finally, to determine which values of $g$ are in fact $\tilde{\sigma}(G)=g$ for a group $G$. The set of such values is called the symmetric crosscap spectrum and there exists a conjecture according to which $g=3$ is the unique positive integer not belonging to the spectrum.

In this paper we deal with the third question. We will study which groups have symmetric crosscap number less than or equal to 17 . First, we will indicate all the results we know and then we will make a study of each group with symmetric crosscap number $g \leq 17$ that has not been studied in detail. Also, results on the spectrum are given. The contents of this paper form part of the doctoral thesis of the author, [3].

\section{Preliminaries}

An NEC group $\Gamma$ is a discrete subgroup of isometries of the hyperbolic plane $\mathcal{H}$, including orientation-reversing elements, with compact quotient $X=\mathcal{H} / \Gamma$. Each NEC group $\Gamma$ has associated a signature [22]:

$$
\sigma(\Gamma)=\left(g, \pm,\left[m_{1}, \ldots, m_{r}\right],\left\{\left(n_{i, 1}, \ldots, n_{i, s_{i}}\right), i=1, \ldots, k\right\}\right)
$$

where $g, k, r, m_{i}, n_{i, j}$ are integers satisfying $g, k, r \geq 0, m_{i} \geq 2, n_{i, j} \geq 2$. We will denote by $[-],(-)$ and $\{-\}$ the cases when $r=0, s_{i}=0$ and $k=0$, respectively.

The signature determines a presentation of $\Gamma$, see [30], by generators $x_{i}(i=1, \ldots, r)$; $e_{i}(i=1, \ldots, k) ; c_{i, j}\left(i=1, \ldots, k ; j=0, \ldots, s_{i}\right) ; a_{i}, b_{i}(i=1, \ldots, g)$ if $\sigma$ has sign ' + '; and $d_{i}(i=1, \ldots, g)$ if $\sigma$ has sign '-'. These generators satisfy the following relations:

$$
x_{i}^{m_{i}}=1 ; \quad c_{i, j-1}^{2}=c_{i, j}^{2}=\left(c_{i, j-1} c_{i, j}\right)^{n_{i, j}}=1 ; \quad e_{i}^{-1} c_{i, 0} e_{i} c_{i, s_{i}}=1
$$

and

$$
\begin{gathered}
\prod_{i=1}^{r} x_{i} \prod_{i=1}^{k} e_{i} \prod_{i=1}^{g}\left(a_{i} b_{i} a_{i}^{-1} b_{i}^{-1}\right)=1 \quad \text { if } \sigma \text { has sign ' }+ \\
\prod_{i=1}^{r} x_{i} \prod_{i=1}^{k} e_{i} \prod_{i=1}^{g} d_{i}^{2}=1 \quad \text { if } \sigma \text { has sign '-' }
\end{gathered}
$$

The isometries $x_{i}$ are elliptic, $e_{i}, a_{i}, b_{i}$ are hyperbolic, $c_{i, j}$ are reflections and $d_{i}$ are glide-reflections. 
Every NEC group $\Gamma$ with signature (2.1) has associated a fundamental region whose area $\mu(\Gamma)$, called area of the group, is:

$$
\mu(\Gamma)=2 \pi\left(\eta g+k-2+\sum_{i=1}^{r}\left(1-\frac{1}{m_{i}}\right)+\frac{1}{2} \sum_{i=1}^{k} \sum_{j=1}^{s_{i}}\left(1-\frac{1}{n_{i, j}}\right)\right),
$$

with $\eta=2$ or 1 depending on the sign ' + ' or ' - ' in the signature. An NEC group with signature (2.1) actually exists if and only if the right-hand side of (2.2) is greater than 0 . We denote by $|\Gamma|$ the expression $\mu(\Gamma) / 2 \pi$ and call it the reduced area of $\Gamma$.

If $\Gamma$ is a subgroup of an NEC group $\Lambda$ of finite index $N$, then also $\Gamma$ is an NEC group and the Riemann-Hurwitz formula holds, $|\Gamma|=N|\Lambda|$.

Let $X$ be a non-orientable Klein surface of topological genus $g \geq 3$ without boundary. Then by [28] there exists an NEC group $\Gamma$ with signature:

$$
\sigma(\Gamma)=(g,-,[-],\{-\}),
$$

such that $X=\mathcal{H} / \Gamma$.

A group $\Gamma$ with this signature is called a surface NEC group. If $G$ acts as an automorphism group of $X=\mathcal{H} / \Gamma$, then there exists another NEC group $\Lambda$ such that $G=\Lambda / \Gamma$. From the Riemann-Hurwitz relation we have $g-2=o(G)|\Lambda|$, where $o(G)$ denotes the order of $G$. Then

$$
\tilde{\sigma}(G) \leq g=2+o(G)|\Lambda|,
$$

and so to obtain the symmetric crosscap number of $G$ is equivalent to find a group $\Lambda$ and an epimorphism $\theta: \Lambda \rightarrow G$, such that $\Gamma=\operatorname{ker} \theta$ is a surface NEC group (and so, without elements with finite order) and $G=\theta\left(\Lambda^{+}\right)$, where $\Lambda^{+}$is the subgroup consisting of the orientation-preserving elements of $\Lambda$, see [28], and minimal $|\Lambda|$.

The groups having symmetric crosscap numbers 1 and 2 have been classified by T. W. Tucker, [29]. The groups of symmetric crosscap number 1 are $C_{n}, D_{n}, A_{4}, S_{4}$ and $A_{5}$. We have two families of groups of symmetric crosscap number $2, C_{2} \times C_{n}, n>2$ even, and $C_{2} \times D_{n}, n$ even. It is known that there exists no group of symmetric crosscap number 3, [23]. The groups with symmetric crosscap number 4 and 5 were obtained in [8].

M. D. E. Conder at a conference in Castro-Urdiales in 2010 announced that using computing software, he had obtained the groups of symmetric crosscap number up to 65 , in terms of their "SmallGroupLibrary" description. The result of this research is available in his webpage, [9]. The list contains the GAP reference of each group, its symmetric crosscap number and the corresponding NEC group $\Lambda$. However, this list gives information neither on the algebraic structure of the groups nor on the epimorphism $\theta$ which determines the action of the NEC group $\Lambda$ over the group $G$. Throughout the paper, we use extensively this fundamental work by Conder, in order to study which are the concerned groups.

For each group $G$ we have described its algebraic structure, its presentation and the corresponding epimorphism, but here we will only show the algebraic structure and its presentation. In the most complicated cases, we will show also the epimorphism. In the presentations we skip the abelian relations. The full details are to be found in [2] and [3]. For groups of order 32 and 64 we use the notation given by Hall and Senior in [20]. The algebraic identification allows us to know the subgroups structure of the involved groups, and this is essential to determine all the groups that act on a surface of a given genus. Along the article $C_{n}, D_{n}, D C_{n}$ and $Q A_{n}$ denote, respectively, the cyclic, dihedral, dicyclic and quasiabelian groups, for more details see [12, 13]. 


\section{Groups of symmetric crosscap number 6 to 9}

In symmetric crosscap number 6 some groups stand out:

1. The group [80, 46]: Coxeter described this group of order 80 in [12], where he named it as $(2,5,5 ; 2)$ with presentation and algebraic structure as shown in the table.

2. The group [160,234]: This group contains the previous one of order 80 . In [12] it is denoted as $(4,5 \mid 2,4)$.

Table 1: Groups of symmetric crosscap number 6 .

\begin{tabular}{l|l|l|l} 
GAP & $G$ & Relations [+ Generators] & Reference \\
\hline \hline$[8,4]$ & $D C_{2} \simeq Q$ & $a^{4}, a^{2} b^{2}, b^{-1} a b a$ & {$[23]$} \\
{$[16,3]$} & $(4,4 \mid 2,2)$ & $a^{4}, b^{4},(a b)^{2},\left(a^{-1} b\right)^{2}$ & {$[15]$} \\
{$[16,6]$} & $Q A_{4}$ & $a^{8}, b^{2}, b a b a^{3}$ & {$[15]$} \\
{$[16,8]$} & $L_{4}$ & $a^{8}, b^{2}, b a b a^{5}$ & {$[15]$} \\
{$[16,13]$} & $\langle 2,2,2\rangle_{2}$ & $a^{2}, b^{2}, c^{2}, a b c a c b, a b c b a c, b c a b a c$ & {$[15]$} \\
{$[16,14]$} & $C_{2} \times C_{2} \times C_{2} \times C_{2}$ & $a^{2}, b^{2}, c^{2}, d^{2}$ & {$[19]$} \\
{$[32,27]$} & $\Gamma_{4} a_{1}$ & $a^{2}, b^{2}, c^{2}, d^{2}, e^{2}, c e c a e$, dedbe & \\
{$[32,43]$} & $\Gamma_{6} a_{1}$ & $a^{8}, b^{2}, c^{2},(a b)^{2}, a c a^{3} c$ & \\
{$[80,46]$} & $(2,5,5 ; 2)$ & $a^{2}, b^{5},(a b)^{5},\left(a^{-1} b^{-1} a b\right)^{2}$ & \\
{$[120,35]$} & $C_{2} \times A_{5}$ & $a^{2},[+(12345),(123)]$ & \\
{$[160,234]$} & $(4,5 \mid 2,4)$ & $a^{4}, b^{5},(a b)^{2},\left(a^{-1} b\right)^{4}$ &
\end{tabular}

Attending to symmetric crosscap number 7, we must analyze the group [72,15], which contains the group of order 36 that appears in the table below (see [26]) and so that the algebraic structure is $\left(\left(C_{2} \times C_{2}\right) \rtimes C_{9}\right) \rtimes C_{2}$. In this case, we are going to give the epimorphism. This group has a presentation given by generators $a, b, c$ and relations $a^{4}=$ $b^{9}=c^{2}=(a c)^{2}=(c b)^{2}=(a b)^{2}=c b^{-1} a b^{-1} a^{-2}=1$. An associated NEC group is $\Lambda$ with signature $(0 ;+;[-] ;\{(2,4,9)\})$ and reduced area $\frac{5}{72}$ and an epimorphism $\theta: \Lambda \rightarrow G$ is

$$
\theta\left(c_{1,0}\right)=c b, \quad \theta\left(c_{1,1}\right)=a c, \quad \theta\left(c_{1,2}\right)=c, \quad \theta\left(c_{1,3}\right)=c b .
$$

The image of $c_{1,1} c_{1,2}$ is the generator $a$, the image of $c_{1,2} c_{1,3}$ is the generator $b$, and finally, $c$ is the image of the element $\left(c_{1,1} c_{1,2}\right)^{2} c_{1,2} c_{1,3}\left(c_{1,1} c_{1,2}\right)^{3} c_{1,2} c_{1,3}$. So we have the generators as images of orientation-preserving elements, and so that the group acts on a non-orientable surface.

For symmetric crosscap number 8 we just have to emphasize the group of order 504 , that is $\operatorname{PSL}(2,8)$, whose symmetric crosscap number was firstly studied in detail by Wendy Hall in [21].

To end this section, we comment some groups with symmetric crosscap number 9 , where we find:

1. The group $[42,1]$, which we call $\langle 7,6,5\rangle$, according to the Coxeter-Moser notation in [13]. It contains $G_{21}$, which is also a group of this symmetric crosscap number, and so that its algebraic structure is $G_{21} \rtimes C_{2}$. Its presentation can be expressed in terms of permutations taking $a=\left(\begin{array}{l}1 \\ 1234567\end{array}\right)$ and $b=\left(\begin{array}{ll}154623\end{array}\right)$. 
Table 2: Groups of symmetric crosscap number 7.

\begin{tabular}{l|l|l|l} 
GAP & $G$ & Relations [+ Generators] & Reference \\
\hline \hline$[12,1]$ & $D C_{3}$ & $a^{6}, a^{3} b^{2}, b^{-1} a b a$ & {$[23]$} \\
{$[24,8]$} & $(4,6 \mid 2,2)$ & $a^{4}, b^{6},(a b)^{2},\left(a^{-1} b\right)^{2}$ & {$[15]$} \\
{$[36,3]$} & $\left(C_{2} \times C_{2}\right) \rtimes C_{9}$ & $a^{2}, b^{2}, c^{9},[a, b], c^{-1} a c b, c^{-1} b c b a$ & \\
{$[72,15]$} & $\left(\left(C_{2} \times C_{2}\right) \rtimes C_{9}\right) \rtimes C_{2}$ & $a^{4}, b^{9}, c^{2},(a c)^{2},(c b)^{2},(a b)^{2}$, & \\
& & $c b^{-1} a b^{-1} a^{-2}$ &
\end{tabular}

Table 3: Groups of symmetric crosscap number 8.

\begin{tabular}{|c|c|c|c|}
\hline GAP & $G$ & Relations [+ Generators] & Reference \\
\hline 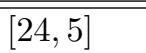 & $C_{4} \times D_{3}$ & $\overline{a^{4}, b^{2}, c^{2},(b c)^{3}}$ & [16] \\
\hline$[24,10]$ & $C_{3} \times D_{4}$ & $a^{3}, b^{2}, c^{2},(b c)^{4}$ & [16] \\
\hline$[48,38]$ & $D_{3} \times D_{4}$ & $a^{2}, b^{2}, c^{2}, d^{2},(a b)^{3},(c d)^{4}$ & [17] \\
\hline$[56,11]$ & $\left(C_{2} \times C_{2} \times C_{2}\right) \rtimes C_{7}$ & $\begin{array}{r}a^{7}, b^{2}, c^{2}, d^{2}, b a d c a^{-1}, c a b a^{-1}, \\
d a c a^{-1}\end{array}$ & \\
\hline$[504,156]$ & $\operatorname{PSL}(2,8)$ & $a^{2}, b^{3},(a b)^{7},\left([a, b]^{4} b\right)^{2}$ & [21] \\
\hline
\end{tabular}

2. The group $[168,42]$ is $\operatorname{PSL}(2,7)$. In this case, the presentation given in the table can be expressed by permutations $b=(234)(576)$ and $a=(123)(456)$ and relations $a^{3}=b^{3}=(a b)^{4}=\left(a^{-1} b\right)^{4}=1$, see [12]. Two more presentations for this group are useful:

(a) $R^{4}, S^{4},(R S)^{2},\left(R^{-1} S\right)^{3}$

(b) $R^{2}, S^{3},(R S)^{7},\left(R^{-1} S^{-1} R S\right)^{4}$

Studying this group, there are actions given by NEC groups with two different signatures:

(i) For an NEC group $\Lambda$ with signature $(0 ;+;[-] ;\{(3,3,4)\})$ and reduced area $\frac{1}{24}$, we take the presentation given by permutations. So an associated epimorphism $\theta: \Lambda \rightarrow G$ is:

$\theta\left(c_{1,0}\right)=\left(b a b a^{2}\right)^{2}, \theta\left(c_{1,1}\right)=\left(a^{2} b\right)^{2}, \theta\left(c_{1,2}\right)=\left(b a^{2}\right)^{2}, \theta\left(c_{1,3}\right)=\left(b a b a^{2}\right)^{2}$

Consider the image of $c_{1,0} c_{1,1}$ and the image of $c_{1,1} c_{1,2}$. Then the image of the element $\left(c_{1,0} c_{1,1}\right)^{2} c_{1,1} c_{1,2} c_{1,0} c_{1,1}\left(c_{1,1} c_{1,2}\right)^{2}$ is $(1543627)$, a permutation of order 7 . This element, together with the elements of order 3 and order 4 , $\theta\left(c_{1,0} c_{1,1}\right)$ and $\theta\left(c_{1,2} c_{1,3}\right)$, generate a group of order 84 at least, but $\operatorname{PSL}(2,7)$ is simple, so it is the full group. So the group $G$ is generated by images of orientation-preserving elements and the group acts on a non-orientable surface.

(ii) For an NEC group $\Lambda$ with signature $(0 ;+;[3] ;\{(4)\})$ and reduced area $\frac{1}{24}$, we use the presentation (b). An associated epimorphism $\theta: \Lambda \rightarrow G$ is:

$$
\theta\left(x_{1}\right)=S, \quad \theta\left(e_{1}\right)=S^{2}, \quad \theta\left(c_{1,0}\right)=R, \quad \theta\left(c_{1,1}\right)=S R S^{-1}
$$


It is clear that $\theta$ is an epimorphism. The element $c_{1,0} x_{1}$ is orientation-reversing, its seventh power is also orientation-reversing and the image of $\left(c_{1,0} x_{1}\right)^{7}$ is the identity element, so the group acts on a non-orientable surface.

3. The group $[336,208]$ has order $336=168 \cdot 2$. Then we can guess its algebraic structure is $\operatorname{PSL}(2,7) \rtimes C_{2}$. We can find a presentation of this group in [10], and an epimorphism $\theta$ does exist. Hence this is the group we are looking for.

Table 4: Groups of symmetric crosscap number 9.

\begin{tabular}{l|l|l|l} 
GAP & $G$ & Relations [+ Generators] & Reference \\
\hline \hline$[21,1]$ & $G_{21}$ & $a^{7}, b^{3}, a b a^{3} b^{-1}$ & {$[15]$} \\
{$[30,1]$} & $C_{5} \times D_{3}$ & $a^{5}, b^{2}, c^{2},(b c)^{3}$ & {$[16]$} \\
{$[30,2]$} & $C_{3} \times D_{5}$ & $a^{3}, b^{2}, c^{2},(b c)^{5}$ & {$[16]$} \\
{$[42,1]$} & $\langle 7,6,5\rangle$ & $a^{7}, b^{6}, b^{-1} a b a^{2}$ & \\
{$[60,8]$} & $D_{3} \times D_{5}$ & $a^{2}, b^{2}, c^{2}, d^{2},(a b)^{3},(c d)^{5}$ & {$[17]$} \\
{$[168,42]$} & $\operatorname{PSL}(2,7)$ & $a^{3}, b^{3},(a b)^{4},\left(a^{-1} b\right)^{4}$ & \\
{$[336,208]$} & $\operatorname{PSL}(2,7) \rtimes C_{2}$ & $a^{3}, b^{8}, c^{2},(a c)^{2},(c b)^{2},(a b)^{2}$, \\
& & $c b^{-1}\left(a b^{-2}\right)^{3} a^{-1}$ &
\end{tabular}

\section{Groups of symmetric crosscap number 10 to 17}

Firstly, we analyze the groups with symmetric crosscap number 10 , where we can find 30 different groups, most of them of order 32,48 and 64 . We just emphasize:

1. For the group $[48,29]$ we use two presentations, the one given in the table (generators $a, b, c$ and relations $\left.a^{2}, b^{3}, c^{3},(b c)^{4},(a b)^{2},(a c)^{2},[b, c](b c)^{2}\right)$ and another one given by generators $R, S$ and relations $R^{8}, S^{3},(R S)^{2}, R^{4} S R^{4} S^{-1}$. For this case, three signatures of NEC groups are given:

(i) For an NEC group $\Lambda$ with signature $(0 ;+;[-] ;\{(2,2,3,3)\})$ and reduced area $\frac{1}{6}$ we take the presentation given in the table and an epimorphism $\theta: \Lambda \rightarrow G$ given by

$$
\theta\left(c_{1,0}\right)=a c, \theta\left(c_{1,1}\right)=(b c)^{2}, \quad \theta\left(c_{1,2}\right)=b a, \quad \theta\left(c_{1,3}\right)=a, \quad \theta\left(c_{1,4}\right)=a c
$$

The group acts on a non-orientable surface, because the image of the element $c_{1,2} c_{1,3}$ is the generator $b$, the image of the element $c_{1,3} c_{1,4}$ is the generator $c$ and the image of the element $c_{1,3} c_{1,1}\left(c_{1,2} c_{1,4}\right)^{2}$ is the generator $a$, so these three images generate the group, and they are images of orientation-preserving elements.

(ii) For an NEC group $\Lambda$ with signature $(0 ;+;[3] ;\{(2,2)\})$ and reduced area $\frac{1}{6}$ we take the second presentation and so an associated epimorphism is $\theta: \Lambda \rightarrow G$ given by

$$
\theta\left(x_{1}\right)=S, \theta\left(e_{1}\right)=S^{-1}, \theta\left(c_{1,0}\right)=R S, \theta\left(c_{1,1}\right)=R^{4}, \theta\left(c_{1,2}\right)=S R
$$


The images of the elements $\left(c_{1,1} c_{1,0} e_{1}\right)^{5}$ and $x_{1}$ are the generators $R$ and $S$ respectively and both are orientation-preserving elements, so it is a group acting on a non-orientable surface.

$$
\theta\left(x_{1}\right)=R S, \theta\left(x_{2}\right)=S, \theta\left(e_{1}\right)=S R^{-1}, \theta\left(c_{1,0}\right)=R^{4}
$$

The quotient gives a non-orientable surface because the images of the elements $x_{1} x_{2}^{2}$ and $x_{2}$ are the generators $R$ and $S$ respectively and both elements are orientation-preserving.

2. The group $[96,70]$ can be expressed in terms of permutations, by means of the generators $a=\left(\begin{array}{ll}1 & 2\end{array}\right)(34)(58)(67)$ and $b=(15)(283647)$.

3. We can find the group $[96,193]$ in [24], called $G_{48}^{*}$, but in the presentation given there, one relation is missing. We have added it, as can be seen in Table 5.

In symmetric crosscap number 11 we have to stand out two things: One is that the presentation of group $[108,15]$ can be expressed in terms of permutations of $S_{18}$ as

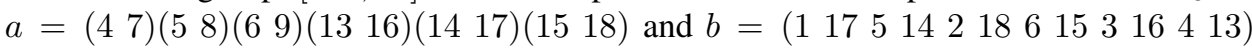
(7 12911810 ); and the other is that the group [108,17] is $G^{3,6,6}$ in the notation of [12].

For symmetric crosscap number 12 and 13, we have nothing to remark.

In symmetric crosscap number 14 we find several groups of order 48 , and the following groups stand out:

1. The presentation of the group $[72,43]$ has been deduced from its algebraic structure $\left(C_{3} \times A_{4}\right) \rtimes C_{2}$. We have taken $d$ as the generator of $C_{2}$ and we have determined how $d$ acts on the other generators.

2. The same argument has been applied to the group [96, 89], where its algebraic structure $\left(D_{2} \times D_{6}\right) \rtimes C_{2}$ determine its presentation. In this case, $e$ is the generator to add. The presentation is given by generators $a, b, c, d, e$ and relations $a^{2}, b^{2}, c^{2}, d^{2}$, $e^{2},(a b)^{2},(c d)^{6}$, eabea, ecdec. Let $\Lambda$ be an associated NEC group with signature $(0 ;+;[-] ;\{(2,2,2,4)\})$ and reduced area $\frac{1}{8}$, so an epimorphism is $\theta: \Lambda \rightarrow G$ given by

$$
\theta\left(c_{1,0}\right)=e, \theta\left(c_{1,1}\right)=b, \theta\left(c_{1,2}\right)=a, \theta\left(c_{1,3}\right)=c, \theta\left(c_{1,4}\right)=e
$$

The elements $c_{1,0}, c_{1,2}, c_{1,3}, c_{1,1}$ and $\left(c_{1,4} c_{1,3}\right)^{2}$ have as images the generators $e, a, c, b, d$ respectively and generate the group. On the other hand the element $\left(c_{1,0} c_{1,2}\right)^{2} c_{1,1}$ has as image the identity element and it is orientation-reversing. Thus, the group acts on a non-orientable surface.

3. The same happens for $[96,115]$ and its algebraic structure is $\left(C_{2} \times D_{12}\right) \rtimes C_{2}$, where $d$ is the generator of $C_{2}$ and so that we have to determine its relations with the other generators.

In symmetric crosscap number 15 , we just note that the group $[1092,25]$ was obtained in [21] by Wendy Hall, who proved that $\operatorname{PSL}(2,13)$ is a group of $84(g-2)$ automorphisms of a surface of genus $g$, and so $g=15$.

Nothing stands out in symmetric crosscap number 16 . But in symmetric crosscap number 17 we have again the same situation that in symmetric crosscap number 14 . For the group $[72,23]$ we have deduced the presentation from its algebraic structure $\left(C_{6} \times D_{3}\right) \rtimes C_{2}$, taking $d$ as the generator of $C_{2}$ and obtaining its action on the other generators. 
Table 5: Groups of symmetric crosscap number 10.

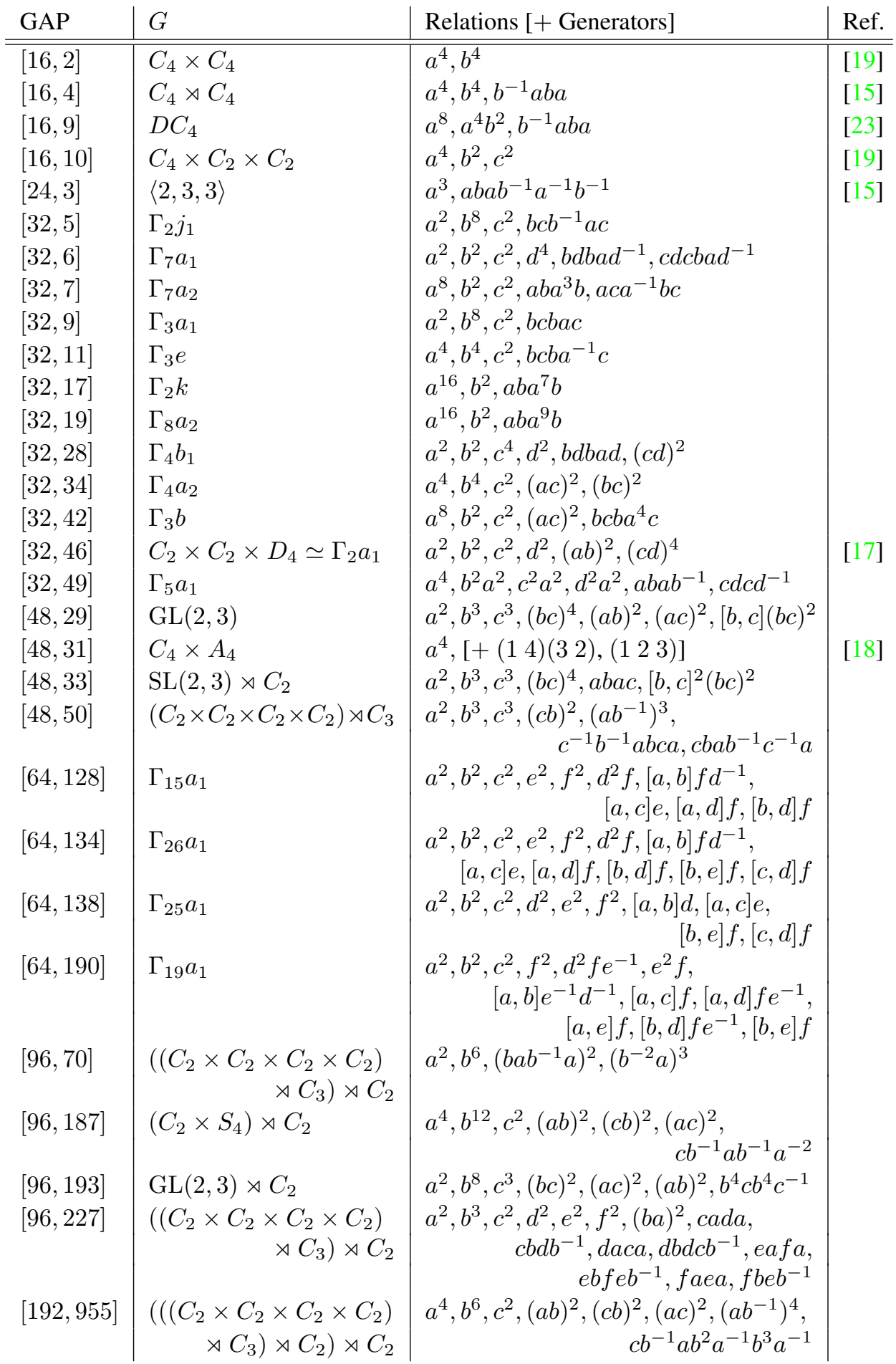


Table 6: Groups of symmetric crosscap number 11.

\begin{tabular}{|c|c|c|c|}
\hline GAP & $G$ & Relations [+ Generators] & \\
\hline$[18,5]$ & $C_{6} \times C_{3}$ & $\overline{a^{6}, b^{3}}$ & \\
\hline$[27,3]$ & $(3,3 \mid 3,3)$ & $a^{3}, b^{3},(b a)^{3},\left(b^{-1} a\right)^{3}$ & \\
\hline$[36,13]$ & $C_{2} \times\left(\left(C_{3} \times C_{3}\right) \rtimes C_{2}\right)$ & $a^{2}, b^{3}, c^{3}, d^{2},(b a)^{2},(c a)^{2}$ & \\
\hline$[54,5]$ & $(2,3,6 ; 3)$ & $a^{3}, b^{6},(a b)^{2},\left(b a^{-1} b\right)^{3}$ & \\
\hline$[54,8]$ & $\left(\left(C_{3} \times C_{3}\right) \rtimes C_{3}\right) \rtimes C_{2}$ & $\begin{array}{l}a^{2}, b^{3}, c^{2},\left(b^{-1} a\right)^{2},(c a)^{3}, \\
\quad\left(b^{-1} c\right)^{2}(b c)^{2},\left(a b^{-1} c\right)^{2} b a c\end{array}$ & \\
\hline$[108,15]$ & $\left(\left(C_{3} \times C_{3}\right) \rtimes C_{3}\right) \rtimes C_{4}$ & $\begin{array}{l}a^{2},\left(b^{-2} a\right)^{3}, b^{-1} a b^{4} a b^{-3} \\
\quad b^{-1} a b a b^{-2} a b a b^{-1} a\end{array}$ & \\
\hline$[108,17]$ & $G^{3,6,6}$ & $a^{2}, b^{2}, c^{2},(a b)^{2},(a c)^{3},(b c)^{6},(a b c)^{6}$ & \\
\hline$[216,87]$ & $\begin{aligned}\left(\left(\left(C_{3} \times C_{3}\right)\right.\right. & \left.\rtimes C_{3}\right) \\
& \left.\rtimes C_{4}\right) \rtimes C_{2}\end{aligned}$ & $\begin{array}{l}a^{4}, b^{6}, c^{2},(a b)^{2},(c b)^{2},(a c)^{2} \\
c\left(b^{-1} a\right)^{3} a\end{array}$ & \\
\hline
\end{tabular}

Table 7: Groups of symmetric crosscap number 12.

\begin{tabular}{l|l|l|l} 
GAP & $G$ & Relations [+ Generators] & Reference \\
\hline \hline$[20,1]$ & $D C_{5}$ & $a^{10}, a^{5} b^{2}, b^{-1} a b a$ & {$[23]$} \\
{$[40,5]$} & $C_{4} \times D_{5}$ & $a^{4}, b^{2}, c^{2},(b c)^{5}$ & {$[16]$} \\
{$[40,8]$} & $\left(C_{10} \times C_{2}\right) \rtimes C_{2}$ & $a^{10}, b^{2},(a b a)^{2},\left(a^{-1} b\right)^{2}(a b)^{2}$ & \\
{$[40,10]$} & $C_{5} \times D_{4}$ & $a^{5}, b^{2}, c^{2},(b c)^{4}$ & {$[16]$} \\
{$[40,12]$} & $C_{2} \times\langle 5,4,2\rangle$ & $a^{5}, b^{4}, b a b^{-1} a^{3}$ & \\
{$[80,39]$} & $D_{5} \times D_{4}$ & $a^{2}, b^{2}, c^{2}, d^{2},(a b)^{5},(c d)^{4}$ & {$[17]$} \\
{$[240,189]$} & $C_{2} \times S_{5}$ & $a^{2},[+(12345),(12)]$ &
\end{tabular}

Table 8: Groups of symmetric crosscap number 13.

\begin{tabular}{l|l|l|l} 
GAP & $G$ & Relations [+ Generators] & Reference \\
\hline \hline$[42,3]$ & $C_{7} \times S_{3}$ & $a^{7},[+(123),(12)]$ & {$[16]$} \\
{$[42,4]$} & $C_{3} \times D_{7}$ & $a^{3}, b^{2}, c^{2},(b c)^{7}$ & {$[16]$} \\
{$[52,3]$} & $C_{13} \rtimes C_{4}$ & $a^{4}, b^{13}, b a b a^{-1}$ & \\
{$[60,9]$} & $C_{5} \times A_{4}$ & $a^{5},[+(123),(14)(23)]$ & {$[18]$} \\
{$[84,8]$} & $D_{3} \times D_{7}$ & $a^{2}, b^{2}, c^{2}, d^{2},(a b)^{3},(c d)^{7}$ & {$[17]$} \\
{$[120,38]$} & $\left(C_{5} \times A_{4}\right) \rtimes C_{2}$ & $a^{4}, b^{15}, c^{2},(a b)^{2},(c b)^{2},(a c)^{2}$, & \\
& \multicolumn{2}{c|}{$c b^{-1} a b^{-1} a^{2}$} &
\end{tabular}


Table 9: Groups of symmetric crosscap number 14 .

\begin{tabular}{|c|c|c|}
\hline GAP & $G$ & Relations [+ Generators $]$ \\
\hline$[16,12]$ & $C_{2} \times Q$ & $a^{4}, a^{2} b^{2}, c^{2}, b^{-1} a b a$ \\
\hline$[24,4]$ & $C_{2} \times D C_{3}$ & $a^{3}, b^{4}, c^{2}, b a b^{-1} a$ \\
\hline$[24,7]$ & $D C_{6}$ & $a^{12}, a^{6} b^{2}, b^{-1} a b a$ \\
\hline$[24,15]$ & $C_{6} \times C_{2} \times C_{2}$ & $a^{6}, b^{2}, c^{2}$ \\
\hline$[32,48]$ & $\Gamma_{2} b$ & $a^{4}, b^{2}, c^{2}, d^{2}, b c b a^{2} c$ \\
\hline$[36,11]$ & $C_{3} \times A_{4}$ & $a^{3},\left[+\left(\begin{array}{ll}1 & 2\end{array}\right)\left(\begin{array}{ll}3 & 4\end{array}\right),\left(\begin{array}{lll}1 & 2 & 3\end{array}\right)\right]$ \\
\hline$[36,12]$ & $C_{6} \times D_{3}$ & $a^{6}, b^{2}, c^{2},(b c)^{3}$ \\
\hline$[48,6]$ & $C_{24} \rtimes C_{2}$ & $a^{24}, b^{2}, b a b a^{13}$ \\
\hline$[48,14]$ & $\left(C_{12} \times C_{2}\right) \rtimes C_{2}$ & $a^{3}, b^{4}, c^{4},(b c)^{2},\left(b^{-1} c\right)^{2}, c^{-1} a c a$ \\
\hline$[48,21]$ & $C_{3} \times(4,4 \mid 2,2)$ & $a^{3}, b^{4}, c^{4},(b c)^{2},\left(b^{-1} c\right)^{2}$ \\
\hline$[48,24]$ & $C_{3} \times Q A_{4}$ & $a^{8}, b^{2}, c^{3}, b a b a^{3}$ \\
\hline$[48,37]$ & $\left(C_{12} \times C_{2}\right) \rtimes C_{2}$ & $\begin{array}{r}a^{3}, b^{2}, c^{2}, d^{2}, d c b c d b, d c b d b c, b d c d b c \\
(b a)^{2},(d a)^{2}\end{array}$ \\
\hline$[48,43]$ & $C_{2} \times\left(\left(C_{6} \times C_{2}\right) \rtimes C_{2}\right)$ & $a^{4}, b^{6}, c^{2},(a b)^{2},\left(a^{-1} b\right)^{2}$ \\
\hline$[48,49]$ & $C_{2} \times C_{2} \times A_{4}$ & $a^{2}, b^{2},\left[+\left(\begin{array}{ll}1 & 2\end{array}\right)\left(\begin{array}{ll}3 & 4\end{array}\right),\left(\begin{array}{lll}1 & 2 & 3\end{array}\right)\right]$ \\
\hline$[48,51]$ & $D_{2} \times D_{6}$ & $a^{2}, b^{2}, c^{2}, d^{2},(a b)^{2},(c d)^{6}$ \\
\hline$[72,42]$ & $C_{3} \times S_{4}$ & $a^{3},\left[+\left(\begin{array}{lll}1 & 2\end{array}\right),\left(\begin{array}{llll}1 & 2 & 3 & 4\end{array}\right)\right]$ \\
\hline$[72,43]$ & $\left(C_{3} \times A_{4}\right) \rtimes C_{2}$ & $\begin{array}{l}a^{3}, b^{2}, c^{3}, d^{2},(d a)^{2},(d c)^{2} \\
\quad\left[+b=\left(\begin{array}{ll}1 & 2\end{array}\right)\left(\begin{array}{ll}3 & 4\end{array}\right), c=\left(\begin{array}{lll}1 & 2 & 3\end{array}\right)\right]\end{array}$ \\
\hline$[72,44]$ & $A_{4} \times S_{3}$ & 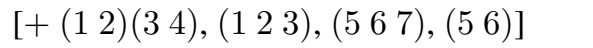 \\
\hline$[72,46]$ & $D_{3} \times D_{6}$ & $a^{2}, b^{2}, c^{2}, d^{2},(a b)^{3},(c d)^{6}$ \\
\hline$[96,89]$ & $\left(D_{2} \times D_{6}\right) \rtimes C_{2}$ & $\begin{array}{r}a^{2}, b^{2}, c^{2}, d^{2}, e^{2},(a b)^{2},(c d)^{6}, \text { eabea }, \text { ecdec }\end{array}$ \\
\hline$[96,115]$ & $\left(C_{2} \times D_{12}\right) \rtimes C_{2}$ & $a^{2}, b^{2}, c^{2}, d^{2},(b c)^{12}, d c b d c$ \\
\hline$[96,226]$ & $C_{2} \times C_{2} \times S_{4}$ & $a^{2}, b^{2},\left[+\left(\begin{array}{llll}1 & 2 & 3 & 4\end{array}\right),\left(\begin{array}{ll}1 & 2\end{array}\right)\right]$ \\
\hline$[144,183]$ & $S_{3} \times S_{4}$ & $\left.\left[+\left(\begin{array}{lll}1 & 2 & 3\end{array}\right),\left(\begin{array}{ll}1 & 2\end{array}\right),\left(\begin{array}{llll}4 & 5 & 6 & 7\end{array}\right),(4) 5\right)\right]$ \\
\hline$[180,19]$ & $A_{5} \times C_{3}$ & $a^{3},[+(132245),(243),(24)(13)]$ \\
\hline$[360,121]$ & $A_{5} \times D_{3}$ & $\begin{array}{l}a^{3}, b^{10}, c^{2},(a b)^{2},(c b)^{2},(a c)^{2} \\
b^{-2} a b^{3} a^{-1} b^{-4} a^{-1} c\end{array}$ \\
\hline
\end{tabular}

Table 10: Groups of symmetric crosscap number 15.

\begin{tabular}{l|l|l|l} 
GAP & $G$ & Relations [+ Generators] & Ref. \\
\hline \hline$[24,1]$ & $\langle-2,2,3\rangle$ & $a^{8}, b^{8},\left(a^{3} b\right)^{3}, a^{2} b^{6}, a^{2}\left(b^{-1} a^{-1}\right)^{3}, b^{2}\left(b^{-1} a^{-1}\right)^{3}$ & [15] \\
{$[39,1]$} & $C_{13} \rtimes C_{3}$ & $a^{3}, b^{13}, b a b^{10} a^{-1}$ & \\
{$[48,15]$} & $\left(C_{3} \times D_{4}\right) \rtimes C_{2}$ & $a^{2}, b^{8}, c^{3},(a b)^{2},(a c)^{2}, b^{-1} c b c$ & \\
{$[78,1]$} & $\left(C_{13} \rtimes C_{3}\right) \rtimes C_{2}$ & $a^{2}, b^{3}, c^{13},(c a)^{2}, c b c^{10} b^{-1}$ & \\
{$[1092,25]$} & $\operatorname{PSL}(2,13)$ & $a^{3}, b^{7}, c^{2},(a b)^{2},(c b)^{2},(a c)^{2}, b^{-1}\left(a b^{-2}\right)^{6} a^{-1} c$ & [21]
\end{tabular}


Table 11: Groups of symmetric crosscap number 16.

\begin{tabular}{l|l|l|l} 
GAP & $G$ & Relations [+ Generators] & Reference \\
\hline \hline$[28,1]$ & $D C_{7}$ & $a^{14}, a^{7} b^{2}, b^{-1} a b a$ & {$[23]$} \\
{$[56,4]$} & $C_{4} \times D_{7}$ & $a^{4}, b^{2}, c^{2},(b c)^{7}$ & {$[16]$} \\
{$[56,7]$} & $\left(C_{14} \times C_{2}\right) \rtimes C_{2}$ & $a^{2}, b^{14},(b a b)^{2},\left(b^{-1} a\right)^{2}(b a)^{2}$ & \\
{$[56,9]$} & $C_{7} \times D_{4}$ & $a^{7}, b^{2}, c^{2},(b c)^{4}$ & {$[16]$} \\
{$[72,16]$} & $C_{2} \times\left(\left(C_{2} \times C_{2}\right) \rtimes C_{9}\right)$ & $a^{9}, b^{2}, c^{2}, d^{2}, b a c b a^{-1}, c a b a^{-1}$ & \\
{$[112,31]$} & $D_{7} \times D_{4}$ & $a^{2}, b^{2}, c^{2}, d^{2},(a b)^{7},(c d)^{4}$ & {$[17]$} \\
{$[144,109]$} & $\left(C_{2} \times\left(\left(C_{2} \times C_{2}\right)\right.\right.$ & $a^{4}, b^{18}, c^{2},(a b)^{2},(c b)^{2},(a c)^{2}$, & \\
& $\left.\left.\times C_{9}\right)\right) \rtimes C_{2}$ & $c b^{-1} a b^{-1} a^{-2}$ &
\end{tabular}

Table 12: Groups of symmetric crosscap number 17.

\begin{tabular}{l|l|l|l} 
GAP & $G$ & Relations [+ Generators] & Ref. \\
\hline \hline$[25,2]$ & $C_{5} \times C_{5}$ & $a^{5}, b^{5}$ & {$[14]$} \\
{$[27,2]$} & $C_{9} \times C_{3}$ & $a^{9}, b^{3}$ & {$[14]$} \\
{$[27,4]$} & $C_{9} \rtimes C_{3}$ & $a^{3}, b^{9}, b a b^{5} a^{-1}$ & {$[15]$} \\
{$[36,6]$} & $C_{3} \times D C_{3}$ & $a^{12}, b^{3}, b a b a^{-1}$ & {$[18]$} \\
{$[50,3]$} & $C_{5} \times D_{5}$ & $a^{5}, b^{2}, c^{2},(b c)^{5}$ & {$[16]$} \\
{$[50,4]$} & $\left(C_{5} \times C_{5}\right) \rtimes C_{2}$ & $a^{2}, b^{5}, c^{5},(b a)^{2},(c a)^{2}$ & \\
{$[54,3]$} & $C_{3} \times D_{9}$ & $a^{3}, b^{2}, c^{2},(b c)^{9}$ & {$[16]$} \\
{$[54,4]$} & $C_{9} \times D_{3}$ & $a^{9}, b^{2}, c^{2},(b c)^{3}$ & {$[16]$} \\
{$[54,6]$} & $\left(C_{9} \rtimes C_{3}\right) \rtimes C_{2}$ & $a^{2}, b^{9}, c^{3},(b a)^{2}, c b^{7} c^{-1} b^{-1}$ & \\
{$[54,7]$} & $\left(C_{9} \times C_{3}\right) \rtimes C_{2}$ & $a^{2}, b^{3}, c^{9},(b a)^{2},(c a)^{2}$ & \\
{$[68,3]$} & $C_{17} \rtimes C_{4}$ & $a^{4}, b^{17}, b a b^{4} a^{-1}$ & \\
{$[72,23]$} & $\left(C_{6} \times D_{3}\right) \rtimes C_{2}$ & $a^{6}, b^{2}, c^{2}, d^{2},(b c)^{3}, b d c b d, d a d a^{3}$ & \\
{$[72,39]$} & $\left(C_{3} \times C_{3}\right) \rtimes C_{8}$ & $a^{8}, b^{3}, c^{3}, b a c a^{-1}, c a b^{-1} a^{-1}$ & \\
{$[100,12]$} & $\left(C_{5} \times C_{5}\right) \rtimes C_{4}$ & $a^{4}, b^{5}, c^{5}, b a b^{3} a^{-1}, c a c^{3} a^{-1}$ & \\
{$[100,13]$} & $D_{5} \times D_{5}$ & $a^{2}, b^{2}, c^{2}, d^{2},(a b)^{5},(c d)^{5}$ & \\
{$[108,16]$} & $D_{3} \times D_{9}$ & $a^{2}, b^{2}, c^{2}, d^{2},(a b)^{3},(c d)^{9}$ & {$[17]$} \\
{$[200,43]$} & $\left(D_{5} \times D_{5}\right) \rtimes C_{2}$ & $a^{4}, b^{10}, c^{2},(a b)^{2},(c b)^{2},(a c)^{2},[a b, b a]$, & \\
{$[360,118]$} & $A_{6}$ & & \\
{$[720,764]$} & $A_{6} \rtimes b_{2}\left(a b^{-3}\right)^{2} a^{-2}$ & \\
& & $a^{3}, b^{8}, c^{2},(a b)^{2},(c b)^{2},(a c)^{2}$, & \\
& & &
\end{tabular}




\section{Groups with symmetric crosscap number $12 k+3$}

Firstly, the strong symmetric genus is the minimum genus of any Riemann surface on which $G$ acts, preserving orientation. For this parameter, there is a group of every strong symmetric genus, [25]. The symmetric genus is the smallest non-negative integer $g$ such that the group $G$ acts faithfully on a closed orientable surface of genus $g$ (not necessarily preserving orientation). For this parameter, the spectrum includes every non-negative integer $g \not \equiv 8$ or $14(\bmod 18)$, and moreover, if a gap occurs at some $g \equiv 8$ or $14(\bmod 18)$, then the prime-power factorization of $g-1$ includes some factor $p^{e} \equiv 5(\bmod 6),[11]$.

In the study of the spectrum of the symmetric crosscap number, the groups with symmetric crosscap number of the form $12 k+3$ are very interesting. It is known that for all $n \neq 12 k+3$, there is a finite group with symmetric crosscap number $n$, see [6]. Conversely, for some values $n=12 k+3$, it is not known whether there exists a group with symmetric crosscap number $n$. So that, we can enunciate some theorems whereby we find infinite families of groups whose symmetric crosscap number is of the type $12 k+3$.

The symmetric crosscap numbers obtained in Theorems 5.1 to 5.5, although of $12 k+3$ form, were already obtained for other groups, as we can see in the proofs. In the case of Theorem 5.6, also these numbers $n$ were already covered, since the group $C_{7(12 k+7)} \rtimes C_{3}$, in the terms of the statement, has symmetric crosscap number $84 k+51$, see [6]. But they are important because they give more examples of groups of this type of $n$, helping us to see how these groups act.

Theorem 5.1. Let $n=12 k+3$ be such that $n-2$ has all its prime factors congruent to $1(\bmod 3)$. Then

$$
C_{12 k+1} \rtimes C_{3} \quad \text { and } \quad\left(C_{12 k+1} \rtimes C_{3}\right) \rtimes C_{2}
$$

have symmetric crosscap number $n$.

Proof. Firstly we have that $C_{12 k+1} \rtimes C_{3}$ has a presentation given by generators $a, b$ such that $a^{3}=b^{12 k+1}=(a b)^{3}=1$. Now let $\Lambda$ be an NEC group with signature $(1 ;-;[3,3]$; $\{-\})$, whose reduced area is $\frac{1}{3}$. We can define an epimorphism $\theta: \Lambda \rightarrow G$ given by

$$
\theta\left(x_{1}\right)=a^{-1}, \theta\left(x_{2}\right)=a b, \theta\left(d_{1}\right)=b^{6 k}
$$

We have that the images of $x_{1}$ and $x_{1} x_{2}$ are the generators $a^{-1}$ and $b$ respectively, and both are preserving-orientation elements, then we have that it is a group that acts on a nonorientable surface. Besides, the NEC group area is minimal ([6]), and so the symmetric crosscap number of $C_{12 k+1} \rtimes C_{3}$ is $n$.

Now we have $\left(C_{12 k+1} \rtimes C_{3}\right) \rtimes C_{2}$ that has a presentation given by generators $a, b, c$ and relations $a^{3}=b^{12 k+1}=c^{2}=(a b)^{3}=1, c a=a c$ and $b c=c b^{-1}$. Now let $\Lambda$ be an NEC group with signature $(0 ;+;[2,3] ;\{(-)\})$, whose reduced area is $\frac{1}{6}$. Therefore, if we define an epimorphism from this NEC group, $\left(C_{12 k+1} \rtimes C_{3}\right) \rtimes C_{2}$ will have symmetric crosscap number less or equal to $n$. We can define an epimorphism $\theta: \Lambda \rightarrow G$ given by

$$
\theta\left(x_{1}\right)=c b, \theta\left(x_{2}\right)=b^{-1} a^{-1}, \theta\left(e_{1}\right)=a c, \theta\left(c_{1,0}\right)=c
$$

We have that the element $c_{1,0}$, the element $e_{1} c_{1,0}$ and the element $c_{1,0} x_{1}$ have as images the generators $c, a$ and $b$ respectively. Besides the element $\left(e_{1} c_{1,0}\right)^{3}$ has as image the identity element and it is orientation-reversing, so we have just proved that the group acts on a non-orientable surface. Because of this epimorphism we can say that $\left(C_{12 k+1} \rtimes C_{3}\right) \rtimes C_{2}$ 
has symmetric crosscap number at most $n$. But since it contains $C_{12 k+1} \rtimes C_{3}$, that has symmetric crosscap number $n, \tilde{\sigma}\left(\left(C_{12 k+1} \rtimes C_{3}\right) \rtimes C_{2}\right)=n$.

Theorem 5.2. Let $n=12 k+3$ be such that $n-2=m^{2}$ is a square. Then:

(i) $(3,3 \mid 3, m)$ has symmetric crosscap number $n$.

(ii) There are two groups with algebraic structure $(3,3 \mid 3, m) \rtimes C_{2}$, namely $(2,3,2 m ; 3)$ and $(2,3,6 ; m)$, that have symmetric crosscap number $n$.

Proof. Firstly we have that the group $(3,3 \mid 3, m)$ of order $3 m^{2}$ has a presentation given by generators $a, b$ and relations $a^{3}=b^{3}=(a b)^{3}=\left(a^{-1} b\right)^{m}=1$. From [15], we know that this group has symmetric crosscap number $m^{2}+2$.

Now we have two groups with algebraic structure $(3,3 \mid 3, m) \rtimes C_{2}$ :

(i) The first one, that is the group $(2,3,2 \mathrm{~m} ; 3)$ in the notation of [12], of order $6 \mathrm{~m}^{2}$, has a presentation given by generators $a, b, c$ and relations $a^{3}=b^{3}=c^{2}=(a b)^{3}=$ $\left(a^{-1} b\right)^{m}=1, c a=a^{2} c$ and $c b=b^{2} c$. Take an NEC group $\Lambda$ with signature $(0 ;+;[2] ;\{(3,3)\})$, that has reduced area $\frac{1}{6}$. We define an epimorphism $\theta: \Lambda \rightarrow G$ given by

$$
\theta\left(x_{1}\right)=c, \theta\left(e_{1}\right)=c, \theta\left(c_{1,0}\right)=a c, \theta\left(c_{1,1}\right)=c b, \quad \theta\left(c_{1,2}\right)=a^{-1} c
$$

We have that $\theta\left(x_{1}\right)=c, \theta\left(c_{1,0} x_{1}\right)=a$ and $\theta\left(x_{1} c_{1,1}\right)=b$, and the element $\left(e_{1} c_{1,0}\right)^{3}$ has as image the identity element and it is orientation-reversing. Thereby we have proved that the group acts on a non-orientable surface. Thereupon we have that this group has symmetric crosscap number at most $m^{2}+2$, but as it contains $(3,3 \mid 3, m)$ that has that symmetric crosscap number $n$, then we have proved that $\tilde{\sigma}((2,3,2 m$; 3)) $=n$.

(ii) The second one, that is the group $(2,3,6 ; m)$ in the notation of [12], also with order $6 m^{2}$, has a presentation given by generators $a, b, c$ and relations $a^{3}=b^{3}=c^{2}=$ $(a b)^{3}=\left(a^{-1} b\right)^{m}=1, a c=c a$ and $b c=c b^{-1}$. For an NEC group $\Lambda$ with signature $(0 ;+;[2,3] ;\{(-)\})$ and reduced area $\frac{1}{6}$, we define an epimorphism $\theta: \Lambda \rightarrow G$ given by

$$
\theta\left(x_{1}\right)=c b, \theta\left(x_{2}\right)=b^{-1} a^{-1}, \theta\left(e_{1}\right)=a c, \theta\left(c_{1,0}\right)=c
$$

We have that the element $c_{1,0}$, the element $e_{1} c_{1,0}$ and the element $c_{1,0} x_{1}$ have as images the generators $c, a$ and $b$ respectively. Besides, the element $\left(e_{1} c_{1,0}\right)^{3}$ has as image the identity element and it is orientation-reversing, so that we have proved that the group acts on a non-orientable surface. So this group has symmetric crosscap number at most $m^{2}+2$, but as it contains $(3,3 \mid 3, m)$, that has that symmetric crosscap number, we have proved that $\tilde{\sigma}((2,3,6 ; m))=n$.

Theorem 5.3. Let $n=12 k+3$ be such that $n-2=m^{2}$ is a square. The symmetric crosscap number of the group $G^{3,6,2 m} \approx\left((3,3 \mid 3, m) \rtimes C_{2}\right) \rtimes C_{2}$ is $n$.

Proof. The group $G^{3,6,2 m}$ of order $12 m^{2}$ has a presentation given by generators $a, b, X, c$ and relations $a^{3}=b^{3}=X^{2}=c^{2}=(a b)^{3}=\left(a^{-1} b\right)^{m}=1, a X=X a^{-1}, b X=$ $X b^{-1}, a c=c a^{-1}$ and $c b=b c$. For an NEC group $\Lambda$ with signature $(0 ;+;[-]$; $\{(2,2,2,3)\})$ we define an epimorphism $\theta: \Lambda \rightarrow G$, given by

$$
\theta\left(c_{1,0}\right)=a X, \theta\left(c_{1.1}\right)=X c, \theta\left(c_{1,2}\right)=c, \theta\left(c_{1,3}\right)=X b, \quad \theta\left(c_{1,4}\right)=a X
$$


We have that the element $c_{1,2}$ has as image the generator $c$, the element $c_{1,1} c_{1,2}$ has as image the generator $X$, the element $c_{1,1} c_{1,2} c_{1,3}$ has as image the generator $b$, and the element $c_{1,4} c_{1,1} c_{1,2}$ has as image the generator $a$. Moreover, the element $\left(c_{1,0} c_{1,1} c_{1,2}\right)^{3}$ has as image the identity element and it is orientation-reversing, so that we have proved that the group acts on a non-orientable surface. The reduced area of the associated NEC group is $\frac{1}{12}$, then we have proved that this group has symmetric crosscap number at most $m^{2}+2$, but as it contains $(2,3,2 m ; 3)$ (see [12]), that has the same symmetric crosscap number, then our group has symmetric crosscap number $n$.

Theorem 5.4. Let $n$ be such that $n=48 k+39$. The symmetric crosscap number of $D C_{3} \times C_{6 k+5}$ and $\left(D C_{3} \times C_{6 k+5}\right) \rtimes C_{2}$ is $n$.

Proof. We have a presentation of the group $\left(D C_{3} \times C_{6 k+5}\right) \rtimes C_{2}$, given by generators $a, b, X, Y$ and relations $a^{4}=b^{3}=X^{6 k+5}=Y^{2}=1, b a=a b^{2}, a Y=Y a^{3}, X Y=$ $Y X^{-1}, Y b=b^{2} Y$ and the rest commute. Let $\Lambda$ be an NEC group with signature $(0 ;+;[-]$; $\{(2,2,3,4(6 k+5))\})$, which has reduced area $\frac{8(6 k+5)-3}{24(6 k+5)}$. So if we can define the adequate epimorphism, we will have that this group has symmetric crosscap number at most $48 k+$ 39 , but as it contains $D C_{3} \times C_{6 k+5}$ that has the same symmetric crosscap number (see [18]), we will be done. Then we take an epimorphism $\theta: \Lambda \rightarrow G$ given by

$$
\theta\left(c_{1,0}\right)=Y X a, \theta\left(c_{1,1}\right)=a^{2}, \theta\left(c_{1,2}\right)=a^{2} Y, \quad \theta\left(c_{1,3}\right)=Y b a^{2}, \theta\left(c_{1,4}\right)=Y X a
$$

We have that the element $c_{1,1} c_{1,2}$ has as image the generator $Y$, the element $c_{1,1} c_{1,2} c_{1,3} c_{1,1}$ has as image the generator $b$. We differentiate between two cases according to the value of $k$ :

(a) If $k$ is even, then we have that the element $\left(c_{1,3} c_{1,4}\right)^{3(6 k+5)+1}$ has as image the generator $X$ and the element $c_{1,1} c_{1,2} c_{1,3} c_{1,1} c_{1,3} c_{1,4}\left(c_{1,3} c_{1,4}\right)^{(6 k+5)-1}$ has as image the generator $a$.

(b) If $k$ is odd, then we have that the element $\left(c_{1,3} c_{1,4}\right)^{(6 k+5)+1}$ has as image the generator $X$ and the element $c_{1,1} c_{1,2} c_{1,3} c_{1,1} c_{1,3} c_{1,4}\left(c_{1,3} c_{1,4}\right)^{3(6 k+5)-1}$ has as image the generator $a$.

So in both cases, we have generated the group with images of elements that preserve the orientation, and thus we have proved that it acts on a non-orientable surface.

Theorem 5.5. Let $n=24 k+15$. The symmetric crosscap number of $C_{3} \rtimes C_{12 k+8}$ and $\left(C_{3} \rtimes C_{12 k+8}\right) \rtimes C_{2}$ is $n$.

Proof. We have a presentation of the group $\left(C_{3} \rtimes C_{12 k+8}\right) \rtimes C_{2}$, given by generators $a, b, c$ and relations $a^{3}=b^{8+12 k}=c^{2}=1, a b=b a^{-1}, c a=a^{-1} c, c b=b^{-1} c$. Let $\Lambda$ be an NEC group with signature $(0 ;+;[-] ;\{(2,2,3,12 k+8)\})$, which has reduced area $\frac{13+24 k}{6(12 k+8)}$. So if we have an epimorphism, we will have that this group has symmetric crosscap number at most $24 k+15$, but as it contains $C_{3} \rtimes C_{12 k+8}$ that has the same symmetric crosscap number (see [6]), we will be done. Then we take an epimorphism $\theta: \Lambda \rightarrow G$ given by

$$
\theta\left(c_{1,0}\right)=c b, \theta\left(c_{1,1}\right)=b^{4+6 k}, \theta\left(c_{1,2}\right)=a c, \theta\left(c_{1,3}\right)=c, \theta\left(c_{1,4}\right)=c b
$$

We have that the element $c_{1,3} c_{1,4}$ has as image the generator $b$, the element $c_{1,2} c_{1,3}$ has as image the generator $a$ and the element $c_{1,0} c_{1,1}\left(c_{1,3} c_{1,4}\right)^{3+6 k}$ has as image the generator $c$. So we have generated the group with images of elements that preserve the orientation, and thus we have proved that it acts on a non-orientable surface. 
Theorem 5.6. Let $n=84 k+51$ be such that $12 k+7$ has all its prime factors congruent to $1(\bmod 3)$. Then the symmetric crosscap number of $C_{4} \times\left(C_{12 k+7} \rtimes C_{3}\right)$ is $n$.

Proof. Firstly we have to indicate, that within the conditions in the statement, there exist groups with order $36 k+21$ with algebraic structure $C_{12 k+7} \rtimes C_{3}$ and with a presentation given by generators $a, b$ and relations $a^{3}=b^{12 k+7}=(a b)^{3}=1$. We call $c$ a generator of $C_{4}$.

Let $\Lambda$ be an NEC group with signature $(0 ;+;[3,12] ;\{(-)\})$ and reduced area $\frac{7}{12}$, and define an associated epimorphism $\theta: \Lambda \rightarrow G$ given by:

$$
\theta\left(x_{1}\right)=b a, \theta\left(x_{2}\right)=a^{-1} c, \theta\left(e_{1}\right)=c^{-1} b^{-1}, \theta\left(c_{1,0}\right)=c^{2}
$$

The element $x_{2}^{9}$ has as image the generator $c$, the element $x_{2}^{8}$ has as image the generator $a$, and the element $x_{1} x_{2}^{4}$ has as image the generator $b$. So we have generated the group with images of orientation-preserving elements and so that it acts on a non-orientable surface. Therefore the symmetric crosscap number of the group will be at most $n$.

On the other hand, the group $C_{12 k+7} \rtimes C_{3}$ can be generated by two elements of order 3 and this condition cannot be lowered. Similarly, an element of order 4 is needed to generate the group $C_{4}$. Hence the area of $\Lambda$ is minimal, because one element of order a multiple of 4 and two elements of order a multiple of an odd number are necessary. Thus, the symmetric crosscap number of our group is $n$.

So that, we need to study some low $k$ to try to find some clues in order to get new numbers in the spectrum. In the previous section we have studied symmetric crosscap number 15 , and in this section we study $12 k+3$ for $k=2,3,4,5$. For each symmetric crosscap number we give the complete list of all groups with that symmetric crosscap number. For that, we have used the Conder's list and the previous theorems to know the algebraic structure and the presentation of some of the groups. It is important to note that all groups $G$ with $\tilde{\sigma}(G)=15,27,39,51$ are provided by the results in the current section.

Table 13: Groups of symmetric crosscap number 27.

\begin{tabular}{l|l|l|l} 
GAP & $G$ & Relations [+ Generators] & Reference \\
\hline \hline$[40,3]$ & $C_{5} \rtimes C_{8}$ & $a^{8}, b^{5}, b a b^{3} a^{-1}$ & {$[3]$} \\
{$[75,2]$} & $(3,3 \mid 3,5)$ & $a^{3}, b^{3},(a b)^{3},\left(a^{-1} b\right)^{5}$ & {$[15]$} \\
{$[150,5]$} & $(2,3,10 ; 3)$ & $a^{3}, b^{3}, c^{2},(a b)^{3},\left(a^{-1} b\right)^{5},(c a)^{2},(c b)^{2}$ & Theorem 5.2 \\
{$[150,6]$} & $(2,3,6 ; 5)$ & $a^{3}, b^{3}, c^{2},(a b)^{3},\left(a^{-1} b\right)^{5},(c b)^{2}$ & Theorem 5.2 \\
{$[300,25]$} & $G^{3,6,10}$ & $a^{3}, b^{3}, c^{2}, d^{2},(a b)^{3},\left(a^{-1} b\right)^{5},(c a)^{2},(c b)^{2}$, & Theorem 5.3 \\
& & $(a d)^{2}$ &
\end{tabular}

From the group [40,3], with symmetric crosscap number 27, and from the group [96, 1], with symmetric crosscap number 63 , other families have been obtained that cover all numbers of the form $24 k+15$ and $60 k+27$, see [6]. So that, it is totally necessary to know the algebraic structure of the groups we have been studying. Another feature of this study is to obtain the groups which are the full automorphism group of a surface of a given genus. This was already done for $g \leq 5$ in [8], for $g=6$ in [4] and for $g=7$ in [5]. 
Table 14: Groups of symmetric crosscap number 39.

\begin{tabular}{l|l|l|l} 
GAP & $G$ & Relations [+ Generators] & Reference \\
\hline \hline$[60,1]$ & $D C_{3} \times C_{5}$ & $a^{4}, b^{3}, c^{5}, b a b a^{-1}$ & {$[18]$} \\
{$[111,1]$} & $C_{37} \rtimes C_{3}$ & $a^{3}, b^{37}, b a b^{27} a^{-1}$ & Theorem 5.1 \\
{$[120,12]$} & $\left(D C_{3} \times C_{5}\right) \rtimes C_{2}$ & $a^{4}, b^{3}, c^{5}, d^{2}, b a b a^{-1},(a d)^{2},(c d)^{2},(d b)^{2}$ & Theorem 5.4 \\
{$[222,1]$} & $\left(C_{37} \rtimes C_{3}\right) \rtimes C_{2}$ & $a^{3}, b^{37}, c^{2}, b a b^{27} a^{-1},(b c)^{2}$ & Theorem 5.1
\end{tabular}

Table 15: Groups of symmetric crosscap number 51.

\begin{tabular}{l|l|l|l} 
GAP & $G$ & Relations [+ Generators] & Reference \\
\hline \hline$[84,2]$ & $C_{4} \times\left(C_{7} \rtimes C_{3}\right)$ & $a^{3}, b^{7}, c^{4}, b a b^{5} a^{-1}$ & Theorem 5.6 \\
{$[147,1]$} & $C_{49} \rtimes C_{3}$ & $a^{3}, b^{49}, b a b^{31} a^{-1}$ & Theorem 5.1 \\
{$[147,5]$} & $(3,3 \mid 3,7)$ & $a^{3}, b^{3},(a b)^{3},\left(a^{-1} b\right)^{7}$ & [15] \\
{$[294,1]$} & $\left(C_{49} \rtimes C_{3}\right) \rtimes C_{2}$ & $a^{3}, b^{49}, c^{2}, b a b^{31} a^{-1},(a c)^{2}$ & Theorem 5.1 \\
{$[294,7]$} & $(2,3,14 ; 3)$ & $a^{3}, b^{3}, c^{2},(a b)^{3},\left(a^{-1} b\right)^{7},(c a)^{2},(c b)^{2}$ & Theorem 5.2 \\
{$[294,14]$} & $(2,3,6 ; 7)$ & $a^{3}, b^{3}, c^{2},(a b)^{3},\left(a^{-1} b\right)^{7},(c b)^{2}$ & Theorem 5.2 \\
{$[588,35]$} & $G^{3,6,14}$ & $a^{3}, b^{3}, c^{2}, d^{2},(a b)^{3},\left(a^{-1} b\right)^{7},(c a)^{2}$, & Theorem 5.3 \\
& & $(c b)^{2},(a d)^{2}$ &
\end{tabular}

Table 16: Groups of symmetric crosscap number 63 .

\begin{tabular}{l|l|l|l} 
GAP & $G$ & Relations [+ Generators] & Reference \\
\hline \hline$[96,1]$ & $C_{3} \rtimes C_{32}$ & $a^{3}, b^{32}, a b a b^{-1}$ & Theorem 5.5 \\
{$[183,1]$} & $C_{61} \rtimes C_{3}$ & $a^{3}, b^{61}, b a b^{48} a^{-1}$ & Theorem 5.1 \\
{$[192,78]$} & $\left(C_{3} \rtimes C_{32}\right) \rtimes C_{2}$ & $a^{3}, b^{32}, c^{2}, a b a b^{-1},(c a)^{2},(c b)^{2}$ & Theorem 5.5 \\
{$[366,1]$} & $\left(C_{61} \rtimes C_{3}\right) \rtimes C_{2}$ & $a^{61}, b^{3}, c^{2}, a b a^{48} b^{-1}, b c b^{-1} c,(a c)^{2}$ & Theorem 5.1
\end{tabular}




\section{References}

[1] N. L. Alling and N. Greenleaf, Foundations of the Theory of Klein Surfaces, volume 219 of Lecture Notes in Mathematics, Springer-Verlag, Berlin, 1971, doi:10.1007/bfb0060987.

[2] A. Bacelo, Los grupos de género imaginario menor que 10, Master's thesis, Universidad Complutense, Madrid, 2013, http://eprints . ucm.es/25482/.

[3] A. Bacelo, Sobre el género imaginario de grupos finitos, Ph.D. thesis, Universidad Complutense, Madrid, 2015, http://eprints.ucm.es/33708/.

[4] A. Bacelo, The full group of automorphisms of non-orientable unbordered Klein surfaces of topological genus 6, Rev. R. Acad. Cienc. Exactas Fís. Nat. Ser. A Math. RACSAM 112 (2018), 391-405, doi:10.1007/s13398-017-0387-6.

[5] A. Bacelo, The full group of automorphisms of non-orientable unbordered Klein surfaces of topological genus 7, Rev. Mat. Complut. 31 (2018), 247-261, doi:10.1007/s13163-017-0245-2.

[6] A. Bacelo, J. J. Etayo and E. Martínez, Filling gaps of the symmetric crosscap spectrum, Moscow Math. J. 17 (2017), 357-369.

[7] E. Bujalance, Cyclic groups of automorphisms of compact nonorientable Klein surfaces without boundary, Pacific J. Math. 109 (1983), 279-289, doi:10.2140/pjm.1983.109.279.

[8] E. Bujalance, J. J. Etayo and E. Martínez, The full group of automorphisms of non-orientable unbordered Klein surfaces of topological genus 3, 4 and 5, Rev. Mat. Complut. 27 (2014), 305-326, doi:10.1007/s13163-013-0121-7.

[9] M. Conder, Groups with symmetric cross-cap number 3 to 65 , https://www math . auckland.ac.nz/ conder/GroupsWithCrosscapNumber3to65.txt.

[10] M. Conder and P. Dobcsányi, Determination of all regular maps of small genus, J. Comb. Theory Ser. B 81 (2001), 224-242, doi:10.1006/jctb.2000.2008.

[11] M. D. E. Conder and T. W. Tucker, The symmetric genus spectrum of finite groups, Ars Math. Contemp. 4 (2011), 271-289, https://amc-journal.eu/index.php/amc/ article/view/127.

[12] H. S. M. Coxeter, The abstract groups $G^{m, n, p}$, Trans. Amer. Math. Soc. 45 (1939), 73-150, doi:10.2307/1990017.

[13] H. S. M. Coxeter and W. O. J. Moser, Generators and Relations for Discrete Groups, volume 14 of Ergebnisse der Mathematik und ihrer Grenzgebiete, Springer-Verlag, Berlin, 4th edition, 1980, doi:10.1007/978-3-662-21943-0.

[14] J. J. Etayo, Sobre grupos de automorfismos de superficies de Klein, Ph.D. thesis, Universidad Complutense, Madrid, 1983, http://eprints.ucm.es/15734/.

[15] J. J. Etayo and E. Martínez, The symmetric crosscap number of the groups of small-order, $J$. Algebra Appl. 12 (2013), 1250164 (16 pages), doi:10.1142/s0219498812501642.

[16] J. J. Etayo Gordejuela and E. Martínez, The symmetric cross-cap number of the groups $C_{m} \times D_{n}$, Proc. Roy. Soc. Edinburgh Sect. A 138 (2008), 1197-1213, doi:10.1017/ s0308210507000169.

[17] J. J. Etayo Gordejuela and E. Martínez, The action of the groups $D_{m} \times D_{n}$ on unbordered Klein surfaces, Rev. R. Acad. Cienc. Exactas Fís. Nat. Ser. A Math. RACSAM 105 (2011), 97-108, doi:10.1007/s13398-011-0007-9.

[18] J. J. Etayo Gordejuela and E. Martínez, The symmetric crosscap number of the families of groups $D C_{3} \times C_{n}$ and $A_{4} \times C_{n}$, Houston J. Math. 38 (2012), 345-358. 
[19] G. Gromadzki, Abelian groups of automorphisms of compact nonorientable Klein surfaces without boundary, Comment. Math. Prace Mat. 28 (1989), 197-217, doi:10.14708/cm.v28i2. 6234.

[20] M. Hall, Jr. and J. K. Senior, The Groups of Order $2^{n}(n \leq 6)$, The Macmillan Co., New York, 1964.

[21] W. Hall, Automorphisms and coverings of Klein surfaces, Ph.D. thesis, University of Southampton, Southampton, 1977, https://eprints.soton.ac.uk/259986/.

[22] A. M. Macbeath, The classification of non-euclidean plane crystallographic groups, Canad. J. Math. 19 (1967), 1192-1205, doi:10.4153/cjm-1967-108-5.

[23] C. L. May, The symmetric crosscap number of a group, Glasg. Math. J. 43 (2001), 399-410, doi:10.1017/s0017089501030038.

[24] C. L. May and J. Zimmerman, Groups of small symmetric genus, Glasgow Math. J. 37 (1995), 115-129, doi:10.1017/s0017089500030457.

[25] C. L. May and J. Zimmerman, There is a group of every strong symmetric genus, Bull. London Math. Soc. 35 (2003), 433-439, doi:10.1112/s0024609303001954.

[26] C. L. May and J. Zimmerman, The groups of symmetric genus $\sigma \leq 8$, Comm. Algebra 36 (2008), 4078-4095, doi:10.1080/00927870802174793.

[27] R. R. Preston, Jr., Fundamental domains and projective structures on compact Klein surfaces, Ph.D. thesis, The University of Texas at Austin, Austin, Texas, 1975, https://search. proquest. com/docview/302777999.

[28] D. Singerman, Automorphisms of compact non-orientable Riemann surfaces, Glasgow Math. J. 12 (1971), 50-59, doi:10.1017/s0017089500001142.

[29] T. W. Tucker, Symmetric embeddings of Cayley graphs in nonorientable surfaces, in: Y. Alavi, G. Chartrand, O. R. Oellermann and A. J. Schwenk (eds.), Graph Theory, Combinatorics, and Applications, Volume 2, John Wiley \& Sons, New York, A Wiley-Interscience Publication, pp. 1105-1120, 1991, proceedings of the Sixth Quadrennial International Conference on the Theory and Applications of Graphs held at Western Michigan University, Kalamazoo, Michigan, May 30 - June 3, 1988.

[30] H. C. Wilkie, On non-Euclidean crystallographic groups, Math. Z. 91 (1966), 87-102, doi: $10.1007 / \mathrm{bf01110157.}$ 